\title{
Sputum microbiota in moderate versus severe patients with COPD
}

\author{
To the Editor:
}

The emergence of new massive sequencing methods has proved revolutionary for the study of complex microbial populations such as the microbiota of the respiratory tract in patients with chronic obstructive pulmonary disease (COPD) [1]. Using this powerful methodology, our objective was to compare the microbiota in two groups of patients with COPD of different severity in order to detect potential microbiological markers that could help to provide a better understanding of the pathogenesis of this disease and the role played by the microbiota in its severity.

The current study recruited nine patients with mild or moderate COPD and 10 patients with severe or very severe COPD in a stable condition (at least 3 months without exacerbation or use of antibiotics for any other reason). Diagnosis and classification of COPD was established according to Global Initiative for Chronic Obstructive Lung Disease (GOLD) recommendations [2].

After DNA extraction from good quality expectorated sputum, quantitative (q)PCR (7500 real time PCR system thermocycler; Life Technologies, Grand Island, NY, USA) was used to quantify the copy number of the 16S rRNA gene in each sample (total bacterial load), yielding a $468 \mathrm{bp}$ amplicon located between nucleotides 340-808 in reference to the 16S rRNA gene of Escherichia coli strain MG1655 (National Center for Biotechnology Information (NCBI) reference sequence: NR_102804.1). In order to compare the results obtained, they were normalised to the number of human cells in the sample, quantified by qPCR, using the human albumin gene [3].

A region measuring $525 \mathrm{bp}$, between position 8 and 533 of the 16S rRNA gene was amplified and pyrosequenced (Roche GS FLX Titanium with Lib-L type microspheres; 454 Life Sciences, Branford, CT, USA). This region comprises the regions of gene hypervariability from V1 to V3 of the $16 \mathrm{~S}$ rRNA gene used to classify bacterial species taxonomically.

The results were analysed bioinformatically using QIIME v16.0 software [4] to filter by quality and size the readings of the sequences obtained by the Roche 454 GS-FLX Titanium. Only sequences measuring between $250 \mathrm{bp}$ and $500 \mathrm{bp}$ with an end-trimming quality greater than 25 analysed in windows of 50 bases were included in the study.

The operational taxonomic units (OTUs) were assigned using the Ribosomal Database Project database for the $16 \mathrm{~S}$ rRNA gene with a bootstrap cut-off of $80 \%$ and only the OTUs representing over $0.5 \%$ of the total sequences of each sample were considered. When all the assignments had been made, the two groups of patients were compared (using ANOVA and the G-test of independence). Other statistical analyses were also carried out: a $\beta$-diversity analysis (principal coordinates analysis (PCoA)) and $\alpha$-diversity analysis (obtaining rarefaction curves and Shannon indexes), using the statistical software R v 2.15.2 with the vegan package (R Project for Statistical Computing. www.r-project.org).

A total of 12893 readings passed the quality filters, of which 6385 sequences belonged to the group of severe patients and 6508 to the moderate group. A mean of 638 sequences per sample were analysed in the severe group and 723 sequences per sample in the moderate group. The most abundant phylum found in the samples was Firmicutes, followed by Proteobacteria, Actinobacteria and Bacteroidetes.

In the $\alpha$-diversity analyses, when the two groups of patients were compared at the genus level, those with moderate disease were seen to exhibit greater bacterial biodiversity. This was reflected in a greater diversity index (Shannon index 4.5, mean of 13 genera per sample) than in those with severe disease (Shannon index 3.1, mean of 7 genera per sample) ( $\mathrm{p}<0.05$, Mann-Whitney U-test), indicating that in severe patients certain microorganisms dominate. Furthermore, the bacterial load was higher in severe patients than in moderate patients (5900 versus 4200 16S rRNA gene copy number per human cell).

Analysis of the sequences obtained in each sample by means of a normalised weighted Unifrac $\beta$-diversity analysis, showed that the majority of samples from moderate patients were found to have a very similar composition of bacterial genera. In fact, a common bacterial core could be seen consisting of the genera Rothia, Prevotella, Veillonella, Fusobacterium, Porphyromonas and Haemophillus. By contrast, the 
composition of bacterial genera in samples from severe patients differed more among themselves and compared to the samples from the other group. The core was displaced by other genera resulting in a decrease in the biodiversity of the respiratory mucosa and a greater dispersion of points in the PCoA analysis.

The association between the genus Actinomyces and severity of COPD is noteworthy. This genus was present in the microbiota of moderate patients (eight out of nine patients) whereas it was less likely to be found in the severe patients (two out of 10 patients) ( $\mathrm{p}<0.01$, ANOVA test). Data are shown in figure 1.

Molecular techniques for characterising the microbiota in patients with COPD have only been used very recently and our study using metagenomics evaluation provides novel information on the microbiota of sputum in COPD patients in a stable clinical situation and important differences between the two study groups were found. The bronchial tree is known to contain a characteristic microbial flora that differs between health and disease [5], and our data also show that there were differences depending on the severity of the disease. Greater bacterial biodiversity was seen in moderate COPD patients, although no such differences were found in previous studies [6].

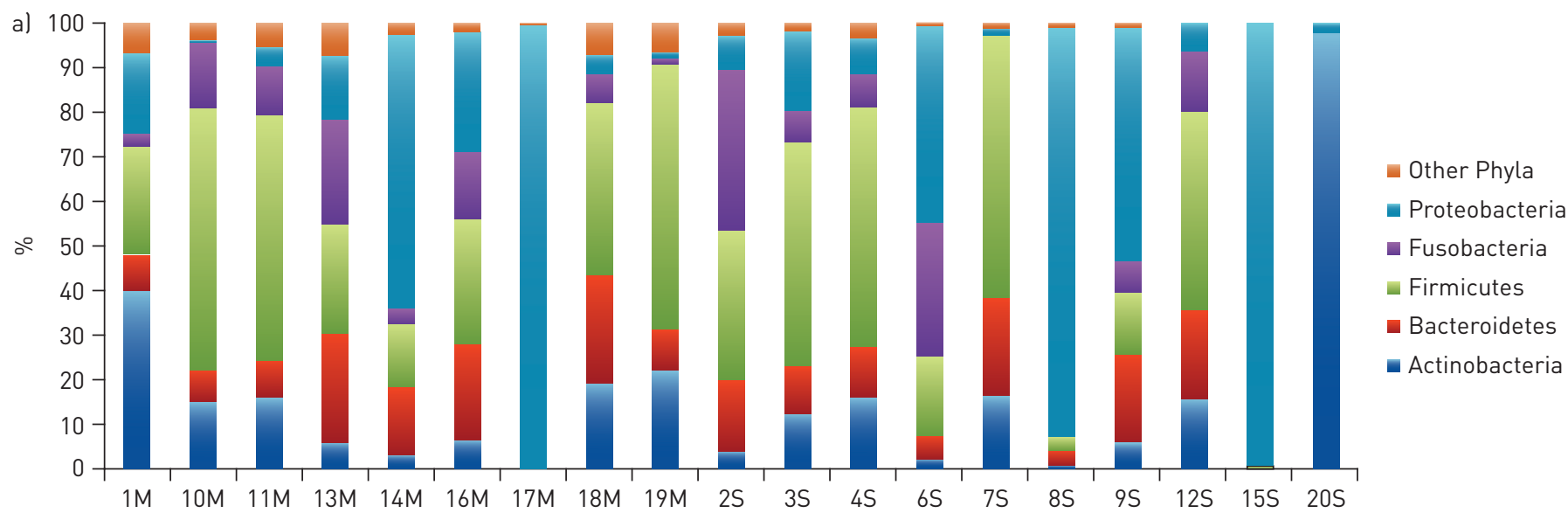

b)

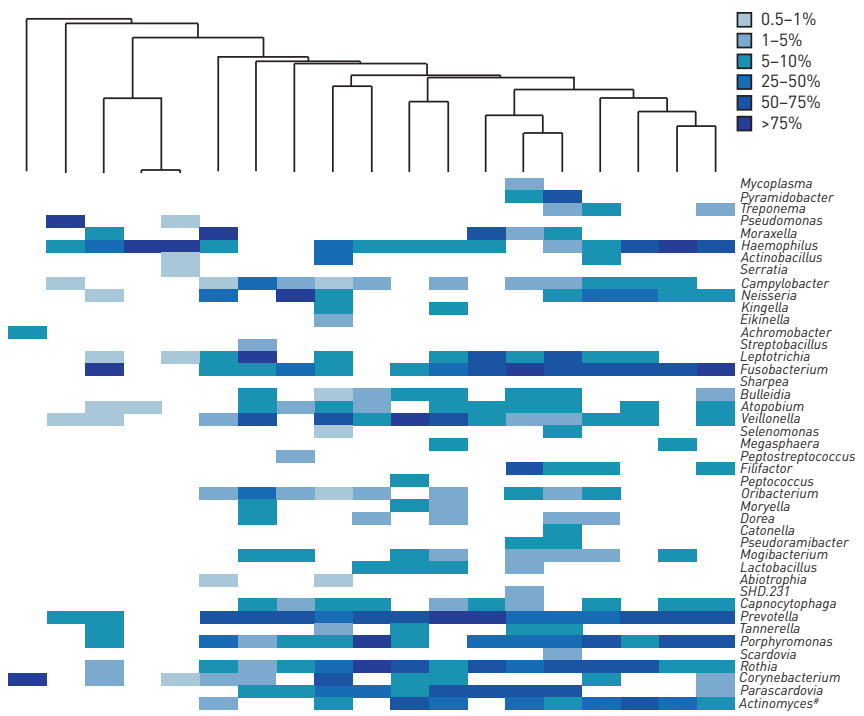

c)

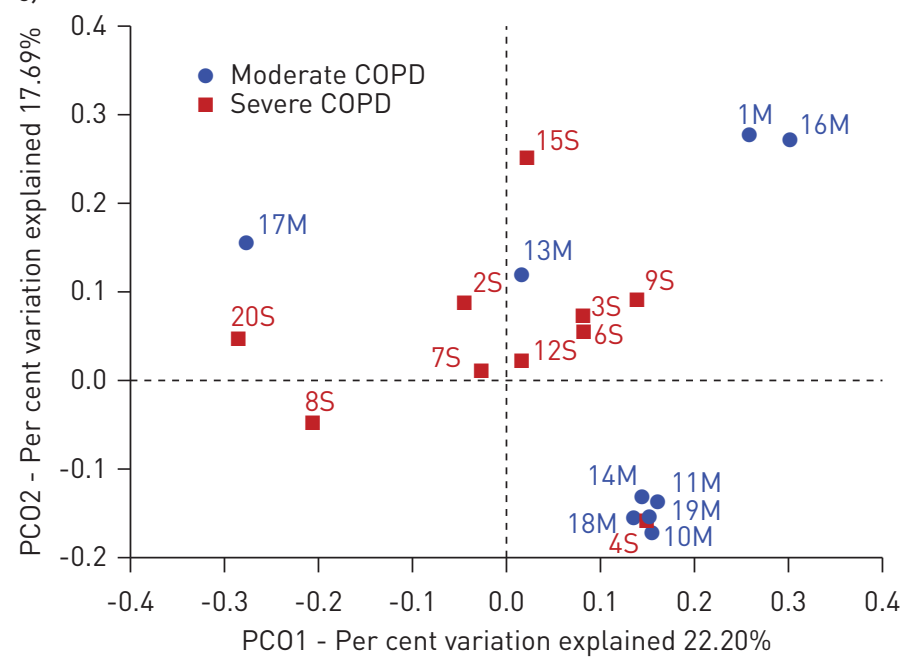

$20 \mathrm{~S} 8 \mathrm{~S}$ 6S 17M 15S 14M 2S 9S 1 M 75 19M 18M 12S 10M 11M 4S 3S 16M 13M

FIGURE 1 a) Bacterial composition at the phylum level in moderate and severe chronic obstructive pulmonary disease (COPD) patients. The chart shows the phylum level classification of $16 \mathrm{~S}$ rDNA sequences identified with at least $80 \%$ bootstrap support in sputum from COPD patients. Samples labelled $1 \mathrm{M}$ to $19 \mathrm{M}$ belong to moderate COPD patients and $2 \mathrm{~S}$ to $20 \mathrm{~S}$ belong to severe COPD patients. b) Bacterial taxonomic profiles by genus and sample clustering by similarity composition. The number of reads assigned to each genus was counted and then the relative abundance was calculated for each genus. Clustering on the $\mathrm{x}$-axis is based on the bacterial composition of each COPD sputum sample; the y-axis shows the relative abundance of each genus. \#: Actinomyces genus. c) Twodimensional principal coordinate (PCO) analysis. Severe and moderate samples clustered separately from each other, samples $10 \mathrm{M}, 11 \mathrm{M}, 14 \mathrm{M}, 18 \mathrm{M}$ and $19 \mathrm{M}$ showed very similar bacterial composition. 
Our study shows that, in addition to different bacterial richness, the absolute quantity of bacteria is greater in more severe pathologies, as indicated by the different absolute bacterial load in the two groups of patients. This complements the previous data which indicate that in the stable state, higher airway bacterial load correlated with more severe airflow limitation and higher inhaled corticosteroid dosage [7]. This correlation with the course of the disease suggests that appropriate antibacterial therapy in colonised patients may be beneficial [8].

The lung is a complex structure composed of cells belonging to all three domains of life on earth, Eukarya, Bacteria and Archaea, as well as their viruses, even in healthy individuals [9]. The microbiome constitutes the last human organ under active research. Like any other organ, the microbiome has physiology and pathology, and an individual's health might be damaged when its collective population structure is altered especially when antibiotics are administered as frequently as they are in these patients $[10,11]$.

In this respect, more detailed studies should be carried out to elucidate the interaction between the microbiome of these patients and repeated exposure to multiple antibiotics in order to achieve a better understanding of the role played by these drugs in altering the structure of the microbiome $[8,12]$. Actinomyces is an indicator that should be evaluated in order to determine its clinical importance in the evolution of these patients since our study found a strong association between the presence or absence of this microorganism and the severity of the clinical condition. Further studies with longer sequences will reveal the Actinomyces species that may be involved, as current pyrosequence reads are only accurate at the genus taxonomic level.

These new methodologies, which are clearly superior to traditional microbiological culture methods, may pave the way for substantially improved diagnosis and treatment of COPD patients since they provide information on the true composition and evolution of the microbiome of the respiratory mucosa depending on the clinical situation of the patient. Thanks to the detection of these changes in the microbiota, it may be possible to initiate therapies at an early stage during disease progression.

$\boldsymbol{0}$

@ERSpublications

Metagenomics evaluation of sputum provides novel data on the microbiota of stable COPD patients http://ow.ly/tMWum

Antonio Galiana ${ }^{1}$, Estefania Aguirre ${ }^{1}$, Juan Carlos Rodriguez ${ }^{2}$, Alex Mira ${ }^{3}$, Miguel Santibañez ${ }^{4}$, Inmaculada Candela $^{5}$, Juana Llavero ${ }^{6}$, Pedro Garcinuño ${ }^{2}$, Francisco López $^{7}$, Montserrat Ruiz ${ }^{1}$, Eduardo Garcia-Pachon ${ }^{8}$ and Gloria Royo ${ }^{1,9}$ ${ }^{1}$ Section of Microbiology, Hospital General Universitario de Elche, Alicante, Spain, ${ }^{2}$ Section of Microbiology, Hospital General Universitario de Alicante, Alicante, Spain, ${ }^{3}$ Centro Superior de Investigación en Salud Pública (CSISP-FISABIO), Valencia, Spain, ${ }^{4}$ Universidad de Cantabria, Santander, Spain, ${ }^{5}$ Centro de Salud de Santa Pola, Santa Pola, Alicante, Spain, ${ }^{6}$ Emergency Unit, Hospital General Universitario de Elche, Elche, Alicante, Spain, ${ }^{7}$ Dept of Internal Medicine, Hospital General Universitario de Elche, Elche, Alicante, Spain, ${ }^{8}$ Section of Respiratory Medicine, Hospital General Universitario de Elche, Alicante, Spain, and ${ }^{9}$ Universidad Miguel Hernández, Elche, Alicante, Spain.

Correspondence: J.C. Rodriguez, Hospital General Universitario de Alicante, Section of Microbiology, Pintor Baeza Street n 10, 03010, Alicante, Spain. E-mail: rodriguez_juadia@gva.es

Received: Nov 042013 | Accepted: Nov 232013 | First published online: Dec 052013

Support statement: This study was supported by: "Ayudas para la realización de trabajos de investigación en materia de atención de media y larga estancia. Agencia Valenciana de Salud. 2010" (MLE 2/10), "Fundación de la Comunidad Valenciana para la investigación biomédica, la docencia y la cooperación internacional y para el desarrollo del Hospital General Universitario de Elche" (FIBELX-CO11/02), "Consejeria de Sanitat. Generalitat Valenciana" (AP-168/11), "Ayuda de investigación de la Fundación Bienvenida Navarro Luciano Trípodi 2010-2011 y 2011-2012" and from the Spanish Ministry of Health (Instituto Salud Carlos III PI12/02209).

Conflict of interest: None declared.

\section{References}

Baquero F, Nombela C. The microbiome as a human organ. Clin Microbiol Infect 2012; 18: Suppl. 4, 2-4.

Rabe KF, Hurd S, Anzueto A, et al. Global strategy for the diagnosis, management, and prevention of chronic obstructive pulmonary disease: GOLD executive summary. Am J Respir Crit Care Med 2007; 176: 532-555.

3 Douek DC, Brenchley JM, Betts MR, et al. HIV preferentially infects HIV-specific CD4 ${ }^{+}$T cells. Nature 2002; 417: 95-98.

4 Caporaso JG, Kuczynski J, Stombaugh J, et al. QIIME allows analysis of high-throughput community sequencing data. Nat Methods 2010; 7: 335-336.

5 Hilty M, Burke C, Pedro H, et al. Disordered microbial communities in asthmatic airways. PLoS ONE 2010; 5: e8578.

6 Pragman AA, Kim HB, Reilly CS, et al. The lung microbiome in moderate and severe chronic obstructive pulmonary disease. PLoS One 2012; 7: e47305. 
7 Garcha DS, Thurston SJ, Patel AR, et al. Changes in prevalence and load of airway bacteria using quantitative PCR in stable and exacerbated COPD. Thorax 2012; 67: 1075-1080.

8 Han MK, Huang YJ, Lipuma JJ, et al. Significance of the microbiome in obstructive lung disease. Thorax 2012; 67: 456-463.

9 Erb-Downward JR, Huffnagle GB, Martinez FJ. The microbiota in respiratory disease. Am J Respir Crit Care Med 2012; 185: 1037-1038.

10 Human Microbiome Project Consortium. Structure, function and diversity of the healthy human microbiome. Nature 2012; 486: 207-214.

Boersma WG. Antibiotics in acute exacerbations of COPD: the good, the bad and the ugly. Eur Respir J2012; 40: 1-3. Sialer S, Adamantia L, Guerrero M, et al. Relation between chronic obstructive pulmonary disease and antibiotics. Curr Infect Dis Rep 2012; 14: 300-307.

\title{
Infantile growth velocity and later asthma/wheeze: GENESIS and the Healthy Growth Study
}

\author{
To the Editor:
}

Accumulating evidence suggests that wheezing illnesses have at least part of their origin in the infantile period, wherein septal restructuring and alveolar formation ensue. Therefore, throughout infancy, genetic and environmental factors could affect bronchial development and future airway function. On this basis, we hypothesised that differential growth velocity, within the first 6 months of life, could be diversely linked to paediatric wheezing illnesses. Establishing such an interaction still eludes us, due to limited and conflicting evidence [1-6] and age-specific differences in bronchial function/structure [3]. We, therefore, opted to investigate a potential correlation in two populations of children of different ages, the Growth, Exercise and Nutrition Epidemiological Study In preSchoolers (GENESIS) study and the Healthy Growth Study cohorts (preschool and late childhood, respectively); thereby, we also appraised any age-definable discrepancies. These cross-sectional studies involved children aged 2-5 years attending nurseries in five Greek counties (GENESIS study) [7] and children aged 9-13 years, attending primary schools in four Greek counties (Healthy Growth Study) [8]. Sampling of nurseries/schools was stratified by parents' educational level and total student population in the respective municipality and their selection was random, as previously described $[7,8]$. By using the parents' educational level as a proxy for socioeconomic level (SEL), we opted to compile a sample that was largely representative of the SEL level of Greek children. Specifically, municipalities were classified in three categories of different SELs i.e. Higher, Medium and Lower (Census 2001 [9]). Subsequently, certain municipalities were randomly selected from each one of these three SEL groups; their number was proportional to the size of each group's preschool/pre-adolescent population. Finally, schools were randomly selected from each municipality; their number was proportional to the municipality's schoolchildren population. Parental consent was acquired and the studies were approved by the Greek Ministry of Education and the Ethics Committee of the Harokopio University, Athens, Greece. Socio-demographic and perinatal data was collected and body fat assessment/anthropometric measurements were conducted during school interviews. The parents provided the researchers with their children's health books wherein (as part of standard practice in Greece) data from routine paediatrician-conducted measurements of infantile weight and length were recorded; this information was used to estimate weightfor-length z-scores based on the World Health Organization (WHO) growth charts. The z-score difference between birth and 6 months of age was used to classify children into the following categories: retarded growth velocity ( $\leqslant 1 \mathrm{z}$-score difference), normal growth velocity $(-1 \leqslant 1 \mathrm{z}$-score difference), and rapid growth velocity ( $>1 \mathrm{z}$-score difference). Potential atopic predisposition of the GENESIS participants was evaluated through the question: "Did a doctor ever tell you that your child has atopic dermatitis?". Asthma outcomes were defined from the International Study of Asthma and Allergies in Childhood (ISAAC) core questionnaire. 1) Current wheeze (did your child have wheezing or whistling in the chest in the past 12 months?). 2) Ever wheeze (did your child have wheezing or whistling in the chest at any point in time?). 3) Asthma ever (did your child ever have asthma diagnosed by a doctor?). Complete data were available from 1668 children in the GENESIS study and 1867 children in the Healthy Growth Study. Univariate logistic regression analyses were conducted and followed by multivariate logistic regression analyses, whereby we adjusted for a wide array of potential confounding/intervening variables (table 1). The 